\title{
Anabases
}

ANABASES Traditions et réceptions de l'Antiquité

19 | 2014

Varia

\section{Sergio AUDANO, Classici lettori di classici. Da Virgilio a Marguerite Yourcenar}

\section{Amedeo Alessandro Raschieri}

\section{OpenEdition}

\section{Journals}

Edizione digitale

URL: http://journals.openedition.org/anabases/4712

DOI: 10.4000/anabases.4712

ISSN: 2256-9421

\section{Editore}

E.R.A.S.M.E.

\section{Edizione cartacea}

Data di pubblicazione: 1 aprile 2014

Paginazione: 330-332

ISSN: 1774-4296

Notizia bibliografica digitale

Amedeo Alessandro Raschieri, « Sergio audano, Classici lettori di classici. Da Virgilio a Marguerite Yourcenar », Anabases [Online], 19 | 2014, Messo online il 01 avril 2014, consultato il 22 septembre 2020. URL : http://journals.openedition.org/anabases/4712 ; DOI : https://doi.org/10.4000/anabases. 4712

Questo documento è stato generato automaticamente il 22 settembre 2020.

(c) Anabases 


\title{
Sergio AUDANo, Classici lettori di classici. Da Virgilio a Marguerite Yourcenar
}

\author{
Amedeo Alessandro Raschieri
}

\section{NOTIZIA}

Sergio AudAno, Classici lettori di classici. Da Virgilio a Marguerite Yourcenar, Foggia, Il

Castello, 2013 («Echo » 8), 312 p.

20 euros / ISBN 978-88-6572-080-6

1 In Italia e all'estero è ben conosciuta l'attività di Sergio Audano come animatore del Centro di Studi «Emanuele Narducci» di Sestri Levante, come organizzatore delle giornate di studio sugli Aspetti della fortuna dell'antico nella cultura europea e come redattore del Notiziario di Antichistica. Grazie a questo volume, che raccoglie alcuni contributi inediti e altri già pubblicati, emergono le sue doti di studioso e profondo conoscitore delle opere antiche, greche e latine, della produzione cristiana e della letteratura italiana. Il libro, infatti, tenta di rivalutare il concetto di classico come processo dinamico di rilettura degli autori precedenti e contiene alcuni approfondimenti intertestuali, spesso nati (come l'autore stesso dichiara) dalla pratica didattica nella scuola superiore. Oltre a tale originalità, Audano dimostra un'ottima conoscenza della bibliografia specialistica (dagli studi più antichi ai contributi più recenti), da cui talvolta prende le distanze, sempre con garbo e buona capacità argomentativa.

2 Nel primo saggio, intitolato Genesi e fortuna di un verso virgiliano : inventas aut qui vitam excoluere per artis (Aen. 6, 663), è analizzato un passo dell'Eneide fondamentale per comprendere il ruolo attribuito alle artes tra poesia didascalica e poema epico. Dopo un approfondimento sulla genesi del verso, posto all'incrocio di influssi lucreziani e 
ciceroniani, lo studioso tratta alcuni momenti della sua ricezione in una catena intertestuale che va da Seneca al motto inciso sulle medaglie del Premio Nobel.

Il secondo saggio, Bruto e il lungo percorso di una sententia virgiliana : Vincet amor patriae laudumque immensa cupido (Aen. 6, 823), si compone di due parti. Nella prima sono trattati i rapporti con i modelli, singoli problemi interpretativi e confronti intertestuali con Cicerone e Valerio Massimo. In particolare, Audano accetta l'ipotesi che, nella caratterizzazione virgiliana di Bruto, sia presente un'intenzionale ambiguità, motivata dalla necessità di problematizzare la sua decisione di uccidere i figli per salvaguardare la res publica. Nella seconda parte è trattata la fortuna di questo verso nei secoli successivi. Agostino, per esempio, contesta la caratterizzazione positiva della figura di Bruto, come presentata nel racconto liviano, e lo descrive come un tiranno. Sono poi delineati i due campi in cui il verso virgiliano è stato utilizzato : la riflessione politica in chiave antitirannica (Tommaso d'Aquino, Machiavelli, Guicciardini) e la polemica letteraria (Bartoli e Alfieri).

4 Il saggio seguente (Leggere l'antico dopo Alfieri : le ambiguità di Massimo d'Azeglio ne I miei ricordi) è dedicato al posto occupato dal classico nell'opera di Massimo d'Azeglio, politico, scrittore e pittore italiano dell'Ottocento, che prese le distanze dall'apprezzamento per la tradizione poetica antica e privilegiò la storiografia di matrice politica e anti-tirannica. Nei suoi scritti, inoltre, emergono il rifiuto della formazione scolastica di tipo gesuitico, la condanna della letteratura classicistica e l'approfondimento sul ruolo di Roma antica nel Risorgimento.

5 I due capitoli seguenti sono dedicati al rapporto tra i classici e due scrittori del Novecento, Carlo Levi e Marguerite Yourcenar. Nel primo (Mito e antimito di Roma nella questione meridionale : riusi virgiliani nel Cristo si è fermato a Eboli di Carlo Levi) Audano dimostra l'influsso delle sezioni virgiliane dedicate ai popoli italici sulla rappresentazione dei contadini lucani, «continuatori di quell'umile Italia agricola e virtuosamente dedita al proprio lavoro" (p. 215) : tale rilettura dell'Eneide è funzionale alla creazione di un antimito contrapposto all'esaltazione di Roma propria del fascismo. Nel secondo saggio (Eraclito e l'eterno ritorno nei Mémoires d'Hadrien di Marguerite Yourcenar) è riconosciuto nei Mémoires l'influsso del pensiero di Eraclito, in particolare della concezione dell'eterno ritorno e del linguaggio del sogno. Grazie alla frequentazione dell'autore antico, la scrittrice può reagire all'insoddisfazione per il pensiero, filosofico e religioso, contemporaneo e valorizzare la capacità di interrogarsi liberamente sulle fondamentali questioni esistenziali.

6 Nell'ultima parte il volume contiene tre brevi note di lettura dedicate al genere consolatorio e al rapporto tra classici latini e musica barocca. Nella prima, intitolata Gli eccessi smaniosi del dolore: riuso epigrammatico di un topos consolatorio (Per la lettura di Marziale 5, 37, 18-19), lo studioso sostiene l'unitarietà formale e retorica dell'epigramma sulla base dell'accorto impiego di alcuni luoghi comuni tipici del genere consolatorio. Nella seconda (Due note su Petrarca e il genere consolatorio) discute l'intreccio tra Cicerone (Rep. 6.14) e Catullo $(3,11-12)$ in un'epistola consolatoria di Petrarca $($ Fam. 14, 3, 6) e ricostruisce il rapporto di un brano del petrarchesco Triumphus Temporis (136-13) con Cicerone (Tusc. 1, 114), Seneca (Epist. 74, 27) e la sententia Sileni. L'ultima nota (Sacra profanis miscere: suggestioni lucreziani nel testo di un mottetto sacro di Nicolò Porpora) è dedicata al testo latino di un mottetto, in cui emerge l'influenza poetica dell'Inno a Venere lucreziano, nonostante il contenuto esplicitamente religioso ed edificante. 
7 Il volume comprende una Nota bibliografica e un Indice selettivo dei passi citati, in cui, a causa di un errore tipografico, risulta errata una parte dei riferimenti interni. In definitiva, il libro è un'ottima dimostrazione della possibilità di incrociare con profitto il mondo della ricerca, la pratica didattica e l'attività di promozione culturale al fine di comprendere sempre meglio, nel dettaglio e nelle questioni generali, il posto occupato dagli autori antichi all'interno della cultura contemporanea.

\section{AUTORI}

\section{AMEDEO ALESSANDRO RASCHIERI}

Università degli Studi di Torino

amedeo.raschieri@alice.it 